\title{
Barriers To and Strategies To Address COVID-19 Testing and Testing Hesitancy: A Rapid Scoping Review
}

\section{Meaghan Sim}

Nova Scotia Health Authority

Hilary A.T. Caldwell

Dalhousie University

\section{Kathryn Stone}

Dalhousie University

\section{Leah Boulos}

Maritime SPOR Support Unit

\section{Ziwa Yu}

Dalhousie University

\section{Gina Agarwal}

McMaster University

\section{Rhiannon Cooper}

McMaster University

Allyson J. Gallant

Dalhousie University

Iwona A. Bielska

McMaster University

Jawad Chishtie

University of Toronto

Janet Curran

Dalhousie University

\section{Andrea Tricco}

Li Ka Shing Knowledge Institute, St. Michael's Hospital

Mark Embrett ( $\nabla$ membrett@dal.ca )

Dalhousie University

\section{Research Article}

Keywords: Covid-19, testing, testing hesitancy, health policy, social determinants of health, equity, coronavirus, scoping review 
Posted Date: October 8th, 2021

DOI: https://doi.org/10.21203/rs.3.rs-944801/v1

License: (c) (i) This work is licensed under a Creative Commons Attribution 4.0 International License. Read Full License 
2 rapid scoping review

3 Mark E. Embrett ${ }^{1,2}$, ORCID: 0000-0002-3969-0219

4 S. Meaghan Sim², ORCID: 0000-0003-4477-3833

5 Hilary A.T. Caldwell ${ }^{3}$, ORCID: 0000-0003-2951-4542

6 Kathryn Stone ${ }^{4}$, ORCID:

7 Leah Boulos ${ }^{5}$, ORCID: 0000-0002-9849-383X

8 Ziwa Yu ${ }^{6}$, ORCID: 0000-0002-7694-5233

9 Gina Agarwal ${ }^{7}$, ORCID: 0000-0002-5691-4675

10 Rhiannon Cooper ${ }^{7}$, ORCID: 0000-0001-9076-5178

11 Allyson J. Gallant AJ ${ }^{1}$, ORCID: 0000-0002-2933-7470

12 Iwona A. Bielska ${ }^{8}$, ORCID: 0000-0002-4186-4871

13 Jawad Chishtie ${ }^{9}$, ORCID: 0000-0001-8650-4469

14 Janet Curran ${ }^{1,6,10}$, ORCID: 0000-0001-9977-0467

15 Andrea Tricco ${ }^{11,12}$, ORCID: 0000-0002-4114-8971

$17{ }^{1}$ Faculty of Health, Dalhousie University, Halifax, NS

$18{ }^{2}$ Research, Innovation \& Discovery, Nova Scotia Health, Halifax, NS

$19{ }^{3}$ Healthy Populations Institute, Dalhousie University, Halifax, NS

$20{ }^{4}$ School of Health and Human Performance, Dalhousie University, Halifax, NS

$21{ }^{5}$ Maritime SPOR Support Unit, Halifax, NS

$22{ }^{6}$ School of Nursing, Dalhousie University, Halifax, NS

23 7Department of Family Medicine, McMaster University, Hamilton, ON

$24{ }^{8}$ Department of Health Research Methods, Evidence and Impact, McMaster

25 University, Hamilton, ON

$26{ }^{9}$ Rehabilitation Sciences Institute, Health Services Outcomes and Evaluation Unit,

27 Faculty of Medicine, University of Toronto

$28{ }^{10}$ IWK Health Centre, Halifax, NS

$29{ }^{11}$ Knowledge Translation Program, Li Ka Shing Knowledge Institute, St. Michael's

30 Hospital, Toronto, Canada

$31{ }^{12}$ Epidemiology Division, Dalla Lana School of Public Health and Institute for

32 Health, Management, and Evaluation, University of Toronto, Toronto, Canada

33

Corresponding Author:

Mark Embrett

membrett@dal.ca

37

Abstract word count: 264

39

$40 \quad$ Full body of text word count: 4497

41 
43 Background: Testing is a foundational component of any COVID-19 management

44 strategy; however, emerging evidence suggests that barriers and hesitancy to

45 COVID-19 testing may affect uptake or participation and often these are multiple

46 and intersecting factors that may vary across population groups. To this end,

47 Health Canada's COVID-19 Testing and Screening Expert Advisory Panel

48 commissioned this rapid review in January 2021 to explore the available

49 evidence in this area. The aim of this rapid review was to identify barriers to

50 COVID-19 testing and strategies used to mitigate these barriers.

52 Methods: Searches (completed January 8 2021) were conducted in MEDLINE,

53 Scopus, medRxiv/bioRxiv, Cochrane and online grey literature sources to identify

54 publications that described barriers and strategies related to COVID-19 testing.

56 Results: From 1294 academic and 97 grey literature search results, 31 academic

57 and 31 grey literature sources were included. Data were extracted from the

58 relevant papers. The most commonly cited barriers were: cost of testing; low

59 health literacy; low trust in the healthcare system; availability and accessibility of

60 testing sites; and stigma and consequences of testing positive. Strategies to

61 mitigate barriers to COVID-19 testing included: free testing; promoting

62 awareness of importance to testing; presenting various testing options and types

63 of testing centres (i.e., drive-thru, walk-up, home testing); providing

64 transportation to testing centres; and offering support for self-isolation (e.g.,

65 salary support or housing).

66 
67 Conclusion: Various barriers to COVID-19 testing and strategies for mitigating

68 these barriers were identified. Further research to test the efficacy of these

69 strategies is needed to better support testing for COVID-19 by addressing testing

70 hesitancy as part of the broader COVID-19 public health response.

71

72 Keywords: Covid-19, testing, testing hesitancy, health policy, social determinants

73 of health, equity, coronavirus, scoping review

74 


\section{Background}

76 COVID-19 is a disease caused by the SARS-CoV-2 virus that was first discovered in

77 Wuhan, China in December 2019 and declared a global pandemic on March 11,

782020 (1). Since then, there have been over 170 million cases worldwide (2).

79 COVID-19 is confirmed by testing, which plays an important role in the find-test-

80 trace-isolate-support cycle to control the spread of COVID-19. Find-test-trace-

81 isolate-support describes testing anyone with symptoms of COVID-19, tracing

82 their contracts, and isolating these individuals until they are no longer contagious

83 (3). While this process can prevent widespread outbreaks of the disease, there

84 are other consequences of widespread testing such as separation from family

85 members while waiting for test results.

86

87 In response to the rapidly growing body of evidence about COVID-19, and the

88 understanding that an find-test-trace-isolate-support public health response is

89 needed, the Canadian Minister of Health commissioned a COVID-19 Testing and

90 Screening Expert Advisory Panel to provide evidence-informed advice to the

91 federal government on science and policy related to to testing and screening(4).

92 The panel consulted with over 80 health experts, public policy experts and

93 members of industry to develop a robust testing approach across Canada. In late

942020 , the authors of this review were approached to conduct a rapid scoping

95 review of COVID-19 testing hesitancy to help the Panel develop guidance about

96 COVID-19 testing.

97 
99 In most jurisdictions, testing is managed by public health authorities. The gold

100 standard for COVID-19 diagnosis is reverse transcription polymerase chain

101 reaction (rRT-PCR) testing from a throat swab or nasopharyngeal swab (5).

102 Diagnosis of COVID-19 allows people and public health authorities to be aware of

103 infections and to take actions to limit the spread to others. Once a test is

104 confirmed positive, it is recommended that the individual and any close contacts

105 isolate for 14 days (6). Despite the population health benefits of COVID-19

106 testing, preliminary evidence shows that COVID-19 testing barriers exist along

107 multiple and intersecting dimensions. Limited access to testing may be the result

108 of testing centre hours, inaccessible environments, locations of testing centres,

109 communication strategies and decisions of how testing is allocated (4). There is

110 also hesitancy related to possible negative consequences of a positive test, such

111 as loss of income or employment or social stigma. The Canadian COVID-19 Expert

112 Panel on Testing and Screening recommends that all jurisdictions implement

113 context-specific strategies to increase testing uptake, such as testing centres in

114 disease hot spots and targeted communication strategies. As the COVID-19

115 pandemic continues to impact communities around the globe, empirical

116 evidence about the barriers to testing and strategies to mitigate barriers is

117 needed to fully implement the find-test-trace-isolate-support model.

119 Conceptual Approach to Organizing Findings

120 To better understand COVID-19 testing hesitancy, we reviewed evidence that

121 aimed to understand individual reasons for hesitating (delaying) to seek

122 healthcare when needed. The 'three delays' model is a prominent model that 
123 separates the decision processes that may influence hesitancy into three

124 decision stages. In other words, the recognition of symptoms and the decision to

125 seek care (planning); finding and reaching an appropriate testing facility and

126 deciding to go through the testing process (process); and receiving adequate care

127 and determining what actions may need to be taken when an outcome is

128 determined (outcomes). The model has primarily been used to understand

129 barriers in access to care in low and middle-income countries by highlighting

130 individual, organizational and system level barriers(7). For present our findings,

131 we adapted the model to outline barriers that influence individuals to seek a

132 COVID-19 test, including knowledge about access, symptoms, etc. (planning), the

133 characteristics of the COVID-19 test itself (process) and consequences of the

134 COVID-19 testing results (outcomes) (Table 1). Strategies to address these

135 barriers were organized in the same manner (Table 2) (8).

137 Review questions

138 Given the importance of COVID-19 testing in the find-test-trace-isolate model, it 139 is necessary to understand factors that prevent people from getting tested and

140 approaches to improve testing uptake. The primary aim of this review is to

141 describe barriers to COVID-19 testing. The secondary aim is to describe effective

142 communication or testing strategies to aid in reducing barriers to COVID-19

143 testing or addressing COVID-19 testing hesitancy. The population, concept and

144 context for this rapid scoping review is: 1) population - persons who are eligible

145 to be tested for COVID-19; 2) concept - COVID-19 testing; and 3) context -

146 testing in any setting. 


\section{Methods}

149 We developed an a priori review protocol according to the research question.

150 We drafted the protocol according to the updated methodological guidance for

151 scoping reviews by Peters et al., adapted for a rapid review by the research

152 team, members of Health Canada, and our teams' information specialist with

153 expertise in designing searches on topics relating to health and economics(9). A

154 rapid review methodology was chosen due to the time constraints upon which

155 this request was needed to inform Health Canada's COVID-19 Expert Panel on

156 Testing and Screening. This review is reported according to the Preferred

157 Reporting Items for Systematic Reviews and Meta-Analyses extension for scoping

158 reviews (PRISMA-ScR), and the search is reported according to the PRISMA

159 extension for literature searches (Tricco et al., 2018).

161 Search strategy

162 An experienced information specialist designed comprehensive search strategies

163 (see Appendix A for detailed information). No study registries were searched. All

164 database searches were executed on January 8, 2021, and results were limited to

165 January 2019-current. Grey literature was retrieved using a combination of

166 targeted website searching and a series of Google queries. Due to the context of

167 the rapid review process, citation searching, and expert consultation were not

168 included in our search methods.

170 Screening \& Data extraction 
171 Covidence, an online tool for conducting various types of reviews

172 (www.covidence.org) was used to review the titles and abstracts for

173 inclusion/exclusion based on the criteria described in Appendix B. Due to the

174 rapid nature of this review, abstracts were reviewed by single reviewers. Next,

175 articles were single screened during full text using the same inclusion/exclusion

176 criteria. Data extraction was completed using the following end-points: (1)

177 country; (2) purpose or aim; (3) study design (if applicable); (4) subgroups and

178 populations of interest; (5) barriers to testing identified; (6) strategies to address

179 barriers or testing hesitancy - either implemented or suggested; and (7) lessons

180 learned, recommendations and outcomes of strategies implemented (if

181 available). The complete data extraction guidelines can be found in Appendix C. 
184 Overview of included studies

185 A total of 1294 unique published articles were identified from the database

186 search. Another 97 grey literature sources were identified. After screening, 61

187 sources were included for data extraction ( $n=30$ academic publications; $n=31$

188 grey literature sources; PRISMA diagram in Appendix C). The majority of studies

189 were from the Unites States $(n=36)$ and the remainder were from Canada $(n=5)$,

190 the United Kingdom or other countries $(n=15)$.

191

192 Findings were categorized in the following two sections: 1) barriers or factors

193 that influence COVID-19 testing and/or testing hesitancy; and 2) strategies to

194 mitigate COVID-19 testing barriers or hesitancy. Each section is then subdivided

195 into sections focusing on the planning, process, and outcomes using the three

196 delays' model conceptual framework (7).

197 Planning barriers

198 The planning stage in the three delays model focuses on factors that influence

199 the decision to seek appropriate medical care once symptoms are noticed or risk

200 of infection is severe enough. In this case, barriers that influence decisions to

201 seek COVID-19 testing found in the literature include the cost of testing, health

202 literacy, trust in the health system, and health status.

204 Cost of testing

205 Cost, often associated with lack of health insurance, was identified as a barrier to

206 seeking COVID-19 testing by eight sources(10-16). Fernando et al. (2020) 
207 described the prohibitive cost of testing, the cost of missing work and

208 transportation costs associated with traveling to and from testing sites as

209 reasons why people did not seek testing. Thunström et al (2020) found that

210 personal financial situations did not affect an individual's willingness to take a

211 free test.

213 Health literacy

214 Health literacy was cited as a barrier to COVID-19by nine sources $(10,17-24)$,

215 Health literacy may refer to limited knowledge of testing, misinformation

216 regarding testing and COVID-19, and/or poor recognition of symptoms.

217 According to the COVID-19 Unified Command Report (2020), low health literacy

218 is associated with risky health behaviours, lower likelihood of seeking treatments

219 and care, and reduced compliance with health-related instructions/guidance,

220 including seeking COVID-19 testing. Misinformation and mixed facts regarding

221 protective measures, transmission, and testing protocols were cited as a reason

222 people do not seek COVID-19 testing $(20,23,24)$. In a longitudinal survey of

223 Australian citizens ( $n=1369$ participants), Bonner et al. (2020) found that not

224 knowing how or where to get tested was a common barrier to testing. Different

225 social groups may be prone to low health literacy preventing testing for various

226 reasons. Undocumented immigrants and foreign workers are often disconnected

227 from the healthcare system and social services, resulting in this group not

228 seeking testing or care when needed (21).

230 Trust in the health system 
231 Low trust in the health system was cited as a barrier to seeking COVID-19 testing

232 by six sources(15,25-29). Due to the nature of the sources, there is limited

233 validated evidence that trust in the health system impacts the likelihood that an

234 individual will or will not seek COVID-19 testing. Distrust in the health system

235 combined with minority status results in decreased access to testing and overall

236 disparities in access to COVID-19 care. Histories of systemic abuse and

237 exploitation of minorities by the medical and research communities, such as the

238 Tuskegee syphilis experiment, were cited by two sources $(25,28)$ as a cause of

239 distrust in the healthcare system.

241 Health status

242 There were two sources that identified current health status as a barrier to

243 testing $(30,31)$. Both sources describe the challenges of encouraging individuals

244 who feel healthy (e.g., asymptomatic) to take COVID-19 tests. Levitt (2020)

245 indicates that refusal for testing among people with self-perceived good health

246 status is one of the greatest barriers to controlling COVID-19; the article further

247 describes the societal costs of healthy individuals not getting tested and suggests

248 ways to incentivize regular testing in this group. Kernberg (2020) determined

249 that among individuals who declined COVID-19 testing, the second most popular

250 reason was confidence that they were not infected with the virus.

\section{Process barriers}

253 The process stage in the three delays model focuses on factors that influence

254 reaching of appropriate medical services, including uncertainty about the 
255 services. In this case, barriers in this delay include the availability and

256 infrastructure of COVID-19 testing sites, including the perceived fear of the

257 testing process.

259 Availability of testing sites

260 Thirteen sources described the availability of testing sites as a process barrier for

261 testing, both in general $(10,25,27,32)$ and in specific communities

$262(18,24,27,29,33)$. Transportation barriers and access to testing centres play a role

263 in accessing COVID-19 testing sites. Among adults surveyed in the United States

264 (cross-sectional, $n=3058$ participants), 15\% reported not being aware of where to

265 get tested or did not have means to travel to get tested (24). In New York City,

266 clusters analysis demonstrated that a lack of availability of testing sites in lower

267 SES communities where there were a higher proportion of positive cases and

268 more severe cases than higher socieoeconomic communities ${ }^{34}$. In comparison,

269 testing sites in Texas were often located in what was described as "whiter"

270 communities ${ }^{21}$ while testing was limited in Black communities, and this was

271 attributed to the inequitable distribution of resources(33). Further, Maxmen et

272 al. (2020) explained that the lack of access to testing was exacerbated in rural

273 and remote communities. Similarly, distance to testing sites increased in rural

274 areas, resulting in a reduction of testing access. Rader and colleagues concluded

275 that geographic barriers to testing exacerbated health inequalities in rural

276 counties. They recommended that geographic accessibility be considered when

277 planning the location of testing sites. Similarly, in remote communities in 
278 Australia, testing availability and timeliness were identified as barriers to testing 279 (34).

281 Infrastructure

282 Two articles identified physical limitations including limited mobility, blindness, 283 low vision, difficulty hearing, communication or understanding information and 284 sensory challenges as barriers to testing $(21,35)$. Further, long wait times for 285 testing and test results were identified as barriers to testing in five studies 286 (24,36-39), where Clipman et al. (2020) reported that $53 \%$ of survey respondents 287 ( $n=3058)$ in the United Stated waited eight or more days for test results, while a 288 drive-through testing in Phoenix had a thirteen hour wait for testing. Thappa et 289 al. (2020) highlighted the discomfort associated with waiting at a facility for 290 results.

292 Discomfort, worry, and distrust were also identified as barriers to testing.

293 Kernberg et al. (2020) found that when offered a monosymptomatic test, 17\% (of 294270 patients) declined, most frequently citing concern for test discomfort.

295 Further, an Australian national survey of 1359 citizens found that pain during 296 testing (11\%) was the most common barrier (Bonner et al., 2020). Four studies 297 reported that the safety of testing sites and worry about infection were barriers 298 to seeking testing(40-43). More specifically, the fears of the safety of test sites 299 were related to physical distancing, isolation, and cleaning practices. Among 300 racialized Americans, Egelko et al. (2020) noted that distrust in the healthcare 301 system and the state was present due to racist treatment that had and continued 
302 to exist within them, and the ongoing role these institutions had in perpetuating

303 health inequities. Similarly, immigrants lacking legal status were also hesitant to

304 receive a test, as there was a fear that the government may use personal

305 information to ensure immigration enforcement (44).

306

307 Outcome barriers

308 The outcomes stage in the three delays model focuses on the consequences once

309 care is received. In this case, the consequences of a positive COVID-19 test.

310 Barriers in this delay include the stigma associated with a positive test and the

311 personal and health costs of a positive test.

313 Stigma

314 Social stigma was identified as a barrier to being tested for COVID-19, as testing

315 may suggest people did not follow public health recommendations (e.g., use of

316 personal protective equipment, physical distancing $(23,45,46)$. Among racialized

317 Americans, there may have been fear of testing due to possible repercussions of

318 testing positive, such as substandard or stigmatized healthcare. One source

319 identified that sex workers in Africa faced stigma and discrimination, and this

320 may have limited their access to contact tracing and COVID-19 testing.

322 Personal and health costs of testing positive

323 Several personal costs were identified as barriers to testing across populations:

324 loss of work and income $(21,32,38,47,48)$, need to quarantine following a

325 positive test (two sources), and fears of deportation among immigrants. 


\section{Planning Strategies}

328 Eliminate cost of testing/Incentivize testing

329 Eighteen sources described strategies intended to address planning barriers.

330 Seven articles overviewed strategies to eliminate the cost of testing, rather than

331 improving convenience of testing(31), although no evidence was provided that

332 these strategies would be effective. Several authors $(19,36,47,49)$, mostly from

333 the United States, proposed improving uptake by eliminating the costs of COVID-

33419 tests, providing incentives to taking tests, including cash benefits, especially

335 for low income or undocumented citizens who may have been afraid to take a

336 test due to immigration status.

338 Promote awareness

339 Doyle et al., (2020) proposed a public information campaign to inform uninsured 340 people in the US that care was available at no charge ${ }^{27}$. They suggested that this

341 would help address misconceptions about the consequences of testing. Capps et

342 al. (2020) recommended that the federal government provide funding to local

343 testing sites to increase uptake of symptomatic individuals ${ }^{28}$. Earnshaw (2020)

344 conducted a cross-sectional survey of 845 adults in the US to examine individual

345 characteristics such as COVID-19 stigma variables (e.g., anticipated stigma and

346 stereotypes), COVID-19 control variables (e.g., knowledge and fear), and

347 sociodemographic characteristics to determine behaviour towards testing ${ }^{49}$.

348 Page et al (2020) suggested funding community and religious organizations to 349 promote awareness around testing and testing sites ${ }^{18}$. 
351 Scientific communication strategy aimed at improving health literacy

352 Two sources suggested that scientists, academics and other experts were able to

353 counteract misinformation about the pandemic, testing and consequences of a

354 positive test to help improve public trust $(22,48)$. Additionally, they suggested

355 building a multi-disciplinary network of academic, community, public, and other

356 partners to understand and address those most impacted by structural

357 inequities, with a focus on testing and tracing. Khaldi et al. (2020) recommended

358 a strategy whereby government bodies provide leadership through initiatives

359 that address misconceptions about the pandemic, testing, outcomes, and other

360 issues that inhibit individuals from testing.

362 Targeted communication strategies aimed at vulnerable populations to improve

363 inequities

364 In a commentary, Thappa et al. (2020) suggested that the Indian government

365 target specific information and education materials that reflected the

366 unconcerned attitude many Indian residents have toward testing. This would

367 require regular updating of information and content. Similarly, in an editorial,

368 Sotgiu et al. (2020) recommended that the government provide a consistent

369 stream of communication that addresses misinformation and provided honest,

370 direct, simple communication from leaders about testing.

372 Egelko et al (2020) proposed that unidirectional messaging was ineffective and

373 would potentially turn individuals away. Instead, they suggested that community 
374 trust needed to be fostered with the aim of making testing an attractive option

375 for all individuals through targeted approaches. Specifically, the authors

376 suggested framing testing as a surveillance method which allows for anonymity

377 instead of as a case-finding method which could be infringing.

379 Various strategies

380 The community council of Tower Hamlet in the U.K. designed a protocol that 381 addressed a local outbreak of COVID-19(50), including strategies for planning,

382 process and outcomes. For planning, they designed a survey and community

383 mapping exercise to gather in-depth insight on support needs. Additionally, a

384 Community Engagement Sub-group had been established to support residents

385 taking part in the national test and trace programme. Initial findings were that

386 community members were more likely to engage in the plan if they were

387 approached by trusted community faith leaders. This helped build trust to

388 convey messages regarding the benefits of testing and contact tracing and

389 responding to data about hotspots or areas of low uptake.

391 Process strategies

393 Availability of testing sites

394 Four articles suggested ways to improve the availability of testing

395 sites $(18,29,51,52)$. Murphy et al., (2020) reported on the implementation of a

396 free testing initiative for Southeastern Pennsylvania Transportation Authority

397 employees offered by the Black Doctors COVID-19 Consortium, as a way to 
398 increase testing for frontline non-hospital workers. Murphy (2021) reported on

399 the same Consortium, where female black doctors provided free tests to

400 underserved communities. The COVID-19 Testing and Contact Tracing Health

401 Equity Guidebook suggested addressing barriers such as transportation by using

402 a mixed approach, including drive-through sites, walk-up sites, mobile screening,

403 and door-to-door screening. Similarly, Maxmen et al., (2020) described a multi-

404 pronged approach to augment access to testing, including testing at physical

405 clinics, youth shelters and by deploying a street medicine team (using a mobile

406 RV) - which was indicated to be useful for contact tracing among individuals

407 experiencing unstable housing.

408

409 Adeniji et al., (2020) suggested allowing self-administered tests and education on

410 how to use them and two others proposed establishing test sites in pharmacies

$411(53,54)^{61}$. Another article described an initiative that used drones to deliver

412 testing kits to remote communities, such as Stoney Nakoda First Nations, Eden

413 Valley, and Big Horn (satellite reserves)(55). Kissam et al., (2020) argued that

414 COVID-19 testing in local areas should shift towards being a component of a

415 comprehensive public health strategy along with contact tracing for those who

416 test positive and additional supports for those required to quarantine/self-

417 isolate.

419 Accessibility

420 The COVID-19 Testing and Contact Tracing Health Equity Guidebook proposed

421 offering bilingual, culturally tailored testing and contact tracing services in 
422 communities with elevated risk. Authors suggested the consideration of

423 processes that include individuals who lack permanent contact information or

424 have unstable housing, and using recognizable locations that are familiar to the

425 community. Maxmen et al., (2020) proposed multiple, accessible testing facilities

426 with providers that reflect community characteristics. Similarly, the Minnesota

427 Department of Health proposed improving accessibility of testing sites for those

428 with physical disabilities by providing signage that is clear, visible, and easy to

429 understand. One article recommended that trusted community leaders and

430 organizations help develop and coordinate testing strategies (56). Similarly,

431 Mitchell et al., (2020) emphasized the need for collaboration and coalitions

432 between academics, laboratories, public health and local healthcare to target

433 vulnerable populations and increase access to tests. Another proposed making

434 testing sites more convenient by providing drive-through testing, which could

435 help to address the fear of infection (57). Galavix et al. (2020) proposed ongoing

436 monitoring and evaluation of testing interventions and their impact on access,

437 where factors such as culture, history, values and needs of minority communities

438 are considered.

439

440 A media article described how Oregon Health \& Science University (OHSU)

441 allotted the first two hours of the testing day for first responders, OHSU health

442 patients, and household members of OHSU health employees (58). To help

443 people understand the process of testing, two healthcare professionals in

444 Saskatchewan agreed to take a COVID-19 test despite being asymptomatic, then 
445 share their lived experience with their patients to decrease stigma and increase

446 education about the discomfort of the test (59).

447

448 Outcome Strategies

449

450 Accommodations for self-isolation \& Support for tracking and tracing

451 One article proposed various strategies to increase testing among minority

452 populations such as increasing the availability of testing sites in minority

453 neighbourhoods and providing cost-free temporary accommodations for self-

454 isolation (60) ${ }^{65}$. Tower Hamlets Council in the United Kingdom linked those

455 isolating to existing supports in the community, such as faith and mutual aid

456 groups and used locally trained and embedded volunteers to support individual

457 residents at every stage of the national test and trace process.

458 
460 This review identified a variety of planning, process, and outcome barriers to

461 testing. Strategies to address testing hesitancy were often well aligned with the

462 testing barriers. For example, the cost of tests was a barrier to accessing testing.

463 Thus, strategies included reducing or eliminating testing costs and/or providing

464 cash incentives for testing, especially for low-income and marginalized groups.

465 To address low health literacy as a barrier to testing, it has been suggested to use

466 scientists, academics, influencers, and other experts to counteract

467 misinformation about testing. Planning barriers to COVID-19 testing included

468 issues with the availability and accessibility of test sites. Appropriate strategies

469 focused on improving the accessibility of testing centres for people with

470 disabilities and multisectoral collaboration within communities while developing

471 the testing centres. Outcome barriers and strategies were least frequently

472 reported on. Outcome barriers that were identified included the social stigma of

473 a positive test if the individual did not follow restrictions, fear of stigmatized

474 healthcare for racialized Americans, and loss of work or income if a positive test

475 was received. The proposed strategy for these barriers was to focus on

476 increasing the availability of testing sites in minority neighbourhoods and the

477 provision of free accommodation for self-isolation.

479 Occasionally, strategies did not align with barriers, presenting gaps in the

480 literature. For instance, the distrust in the healthcare system, experienced

481 especially by racialized Americans, though identified as a planning, process, and

482 outcome barrier in a variety of capacities, was not directly addressed by any 
483 strategy. Although process strategies did include culturally tailored testing

484 services and interventions that considered culture, history, and values of

485 minority communities, strategies were vague or localised geographically and

486 therefore not directly designed to target healthcare system distrust. More

487 research should be conducted to understand and implement tactical solutions

488 that address the root causes of healthcare system distrust.

489

490 Mukattash et al. (2020) indicated that pharmacists could provide testing in their

491 communities in Jordan with appropriate training. Further studies found in the

492 broader literature discussed the crucial role that pharmacists played in

493 community health and the response to COVID-19 (61,62). Moreover, a pilot

494 study in Alberta, where COVID-19 testing was made available in 20 pharmacies,

495 quickly expanded to 168 pharmacies and counting (63), though Canada had yet

496 to see widespread testing in pharmacies compared to that in the United States

$497(64,65)$. Testing in pharmacies could be an important consideration for

498 policymakers in Canada.

500 Strengths and Limitations

501 Strengths of this review include our comprehensive search strategy and inclusion

502 of grey literature. As the COVID-19 pandemic continues to evolve, it was

503 necessary to include the most up-to-date, current information. A number of

504 limitations of this review were identified. First, the majority of sources (39) were

505 opinion pieces (i.e., commentaries, perspectives, media articles). Two sources

506 were guidelines to prevent stigma and carrying out contact tracing, and twenty- 
507 one articles collected or analyzed primary or secondary data. These findings

508 demonstrate a lack of experimental and observational designs to better

509 understand the development of barriers to COVID-19 testing and to direct the

510 impact of strategies. There is a need for both types of studies to determine the

511 extent to which a barrier inhibits COVID-19 test seeking behaviour and the

512 effectiveness of strategies to improve the uptake of COVID-19 testing or address

513 testing hesitancy. Further, the majority of publications were from the United

514 States, which limits our understanding of testing barriers in other areas of the

515 world.

516

517 Conclusion

518 This review intended to identify and summarize the best available evidence to

519 inform Canada's COVID-19 Expert Panel on Testing. The impact or effectiveness

520 of strategies to address COVID-19 testing barriers or testing hesitancy is largely

521 unknown. The information found within this review provides an evidentiary basis

522 to suggest that multi-pronged approaches to addressing barriers are being both

523 suggested and implemented. The use of implementation science frameworks

524 may be a means of developing, evaluating, and refining approaches to

525 addressing COVID-19 testing barriers and hesitancy.

526

527

528 


\section{References}

530 1. Adebisi, Y. A., Alaran, A. J., Akinokun, R. T., Micheal, A. I., Ilesanmi, E. B., \&

531 Lucero-Prisno, D. E. (2020). Sex Workers Should not Be Forgotten in Africa's COVID-19 Response. The American Journal of Tropical Medicine and Hygiene, 103(5 PG-1780-1782), 1780-1782. https://doi.org/https://dx.doi.org/10.4269/ajtmh.20-1045

2. Al-Quteimat, O. M., \& Amer, A. M. (2021). SARS-CoV-2 outbreak: How can pharmacists help? Research in Social and Administrative Pharmacy, 17(2), 480-482. https://doi.org/https://doi.org/10.1016/j.sapharm.2020.03.018

3. Amariles, P., Ledezma-Morales, M., Salazar-Ospina, A., \& Hincapié-García,

J. A. (2021). How to link patients with suspicious COVID-19 to health system from the community pharmacies? A route proposal. Research in Social and Administrative Pharmacy, 17(1), 1988-1989. https://doi.org/https://doi.org/10.1016/j.sapharm.2020.03.007

4. Babych, S. (2020). Research project connecting remote communities with COVID-19 test kits using drones. Calgary Herald, (PG-1-6), 1-6. Retrieved from NS -

5. Bartlett, J., Boston, R., \& Journal, B. (2021). Cost of coronavirus test is a travel barrier for some. (PG-1-7), 1-7. Retrieved from NS -

6. Bonner, C., Batcup, C., Pickles, K., Dodd, R., Copp, T., Cornell, S., ... McCaffery, K. (2020). Behavioural barriers to COVID-19 testing. SSRN Electronic Journal, 1-24.

7. Capps, R., \& Gelatt, J. (2020). Barriers to COVID-19 testing and treatment: Immigrants without health insurance coverage in the United States. 

s-FS_Final.pdf NS -

8. Clipman, S. J., Wesolowski, A., Mehta, S. H., Agarwal, S., Cobey, S. E., Cummings, D. A. T., ... Solomon, S. S. (2020). SARS-CoV-2 Testing in Florida, Illinois, and Maryland: Access and Barriers. MedRxiv : The Preprint Server for Health Sciences, (101767986 PG-). https://doi.org/https://dx.doi.org/10.1101/2020.12.23.20248789

9. Command, C.-19 U. (2020). COVID-19 Testing and Contact Tracing Health Equity Guidebook. Retrieved from NS and contextual factors in New York City. Spatial and Spatio-Temporal Epidemiology, 34(100355). https://doi.org/https://dx.doi.org/10.1016/j.sste.2020.100355 Retrieved from https://www.towerhamlets.gov.uk/Ignl/health_social_care/health_and_ medical_advice/Coronavirus/Local_outbreak_plan/Community_Engagem ent_for_Test_and_Trace.aspx NS -

12. Diagnostics, Q. (2020). New Quest Diagnostics Health Trends ${ }^{T M}$ Survey Reveals COVID-19 Testing Hesitancy Among Americans, With 3 of 4 Avoiding a Test When They Believed They Needed One. (PG-). Retrieved from NS -

13. Dodds, C., \& Fakoya, I. (2020). Covid-19: ensuring equality of access to 
testing for ethnic minorities. BMJ (Clinical Research Ed.), 369(8900488, bmj, 101090866 PG-m2122), m2122. https://doi.org/https://dx.doi.org/10.1136/bmj.m2122

14. Doyle, C., Byrne, K., Fleming, S., Griffiths, C., Horan, P., \& Keenan, P. M. (2016). Enhancing the experience of people with intellectual disabilities who access health care. Learning Disability Practice, 19(6), 33-38. https://doi.org/10.7748/ldp.2016.e1752

15. Doyle, S. (2020). Migrant workers falling through cracks in health care coverage. CMAJ : Canadian Medical Association Journal = Journal de I'Association Medicale Canadienne, 192(PG-E819-E820), E819-E820. https://doi.org/10.1503/cmaj.1095882

16. Dryden-Peterson, Sarah, Dahya, N., \& Adelman, E. (2017). Pathways to Educational Success Among Refugees: Connecting Locally and Globally Situated Resources. American Educational Research Journal, 54(6), 10111047. https://doi.org/10.3102/0002831217714321

17. Dryden-Peterson, Scott, Velasquez, G. E., Stopka, T. J., Davey, S., Lockman, S., \& Ojikutu, B. (2020). SARS-CoV-2 Testing Disparities in Massachusetts. MedRxiv : The Preprint Server for Health Sciences, (101767986 PG-). https://doi.org/https://dx.doi.org/10.1101/2020.11.02.20224469 Testing. Stigma and Health, 5(PG-390-393), 390-393. 
601

602

603

604

605

606

607

608

609

610

611

612

613

614

615

616

617

618

619

620

621

622

623

624

19. Egelko, A., Arnaout, L., Garoon, J., Streed, C., \& Berger, Z. (2020). “Do I Have to Be Tested?": Understanding Reluctance to Be Screened for COVID-19. American Journal of Public Health, 110(12 PG-1769-1771), 1769-1771.

https://doi.org/https://dx.doi.org/10.2105/AJPH.2020.305964

20. Evans, M. K. (2020). Covid's Color Line - Infectious Disease, Inequity, and Racial Justice. New England Journal of Medicine, 383(PG-408-410), 408410. https://doi.org/10.1056/NEJMp2019445

21. Feldman, B. N. (2021). Black Doctors Work to Make Coronavirus Testing More Equitable. (PG-1-8), 1-8. Retrieved from NS -

22. Fernando, C., \& Thompson, C. (2020). Inequality ' baked into ' virus testing access as cases surge. AP News, (PG-5-11), 5-11. Retrieved from NS -

23. Fleming, N. (2020). Coronavirus misinformation, and how scientists can help to fight it. Nature. Retrieved from https://search.proquest.com/docview/2406985800 accountid=26724 http://sfx.library.cdc.gov/cdc/?url_ver=Z39.882004\&rft_val_fmt=info:ofi/fmt:kev:mtx:journal\&genre=article\&sid=ProQ: ProQ\%3Ahealthcompleteshell\&atitle=FIGHT+CORONAVIRUS+MISINFORM ATION\&title $=$ Nat

24. Fusco, F. M., Sangiovanni, V., Tiberio, C., Papa, N., Atripaldi, L., \& Esposito, V. (2020). Persons living with HIV may be reluctant to access to COVID-19 testing services: data from “D. Cotugno” Hospital, Naples, Southern Italy. AIDS (London, England), 34(14 PG-2151-2152), 2151- 
25. Galaviz, K. I., Breland, J. Y., Sanders, M., Breathett, K., Cerezo, A., Gil, O., ... Essien, U. R. (2020). Implementation Science to Address Health Disparities During the Coronavirus Pandemic. Health Equity, 4(1 PG-463467), 463-467. https://doi.org/https://dx.doi.org/10.1089/heq.2020.0044

26. Gebhart, F. (2020). States slowly ease barriers to pharmacist-provided covid-19 testing. Formulary, 164, 9.

27. Government of Canada. (2021). Priority strategies to optimize testing and screening for COVID-19 in Canada: Report. Retrieved May 25, 2021, from https://www.canada.ca/en/health-canada/services/drugs-healthproducts/covid19-industry/medical-devices/testing-screening-advisorypanel/reports-summaries/priority-strategies.html Review of COVID-19 (Coronavirus Disease-2019) Diagnosis, Treatments and Prevention. Eurasian Journal Of Medicine And Oncology, 4(2), 116125. Retrieved from https://dx.doi.org/10.14744/ejmo.2020.90853 29. Health, M. D. of. (2021). Improving COVID-19 Testing for People with Disabilities and Unique Health Needs. Retrieved from https://www.health.state.mn.us/communities/equity/about/c19testing.h tml NS - 
address inequities in the COVID-19 response. The Lancet. Infectious Diseases, (101130150 PG-). https://doi.org/https://dx.doi.org/10.1016/S1473-3099(20)30859-8 Testing Is Essential For Health Equity. Health Affairs Blog, (PG-1-8), 1-8. https://doi.org/10.1377/hblog20200611.868893

32. Ibarra, A. (2021). For Black and Latino Communities, Trust Is an Issue for Coronavirus Testing. pp. 1-7. Retrieved from NS -

33. Jacobson, T. A., Smith, L. E., Hirschhorn, L. R., \& Huffman, M. D. (2020). Using implementation science to mitigate worsening health inequities in https://doi.org/https://dx.doi.org/10.1097/AOG.0000000000004127 

from http://krinstitute.org/assets/contentMS/img/template/editor/20200325_ Articles_Covid_FW_v3.pdf

37. Kissam, E. (2020). The impact of the COVID-19 pandemic on California farmworkers: Better local data collection and reporting will improve strategic response. Statistical Journal of the IAOS, 36(4 PG-867-898),

38. Lan, R., Sujanto, R., Lu, K., He, Z., Zhang, C. J. P., \& Ming, W.-K. (2020). Perceived Effectiveness, Safety, and Attitudes Toward the Use of Nucleic Tests of SARS-CoV-2 Among Clinicians and General Public in China. Frontiers in Public Health, 8(101616579 PG-599862), 599862. https://doi.org/https://dx.doi.org/10.3389/fpubh.2020.599862

39. Lefman, S. H., \& Prittie, J. E. (2019). Psychogenic stress in hospitalized veterinary patients: Causation, implications, and therapies. Journal of Veterinary Emergency and Critical Care (San Antonio, Tex. : 2001), 29(2), 107-120. https://doi.org/10.1111/vec.12821

40. Li, S., Feng, B., Liao, W., \& Pan, W. (2020). Internet Use, Risk Awareness, and Demographic Characteristics Associated With Engagement in Preventive Behaviors and Testing: Cross-Sectional Survey on COVID-19 in the United States. Journal of Medical Internet Research, 22(6 PG-e19782), e19782. https://doi.org/https://dx.doi.org/10.2196/19782

41. Loyola University. (2021). COVID Equity Response Collaborative: Loyola (CERCL). Retrieved from http://www.luc.edu/parkinson/cercl/ NS - 
42. Mahase, E. (2020). Covid-19: Mental health consequences of pandemic need urgent research, paper advises. 1515(April), 1-2. https://doi.org/10.1136/bmj.m1515

43. Martin, T., \& Martin, A. (2021, June 12). Sask . Health Authority seeks to' help the hardest hit. Nature, (0410462 PG-). https://doi.org/https://dx.doi.org/10.1038/d41586-020-01781-z

45. Mukattash, T. L., Jarab, A. S., Abu-Farha, R. K., Nusair, M., Mukattash, I. L., Obaidat, R. M., ... Basheti, I. (2020). Willingness and readiness to test for COVID-19; A qualitative exploration of community pharmacists. International Journal of Clinical Practice, 74(12 PG-e13620), e13620. https://doi.org/https://dx.doi.org/10.1111/ijcp.13620

46. Murphy, D. (2021). Black Doctors will test SEPTA employees for COVID-19 at stations. The Pulse, pp. 1-8.

47. News, B. (2021). Black women doctors give free COVID-19 test kits to underserved areas. National News, (PG-). Retrieved from NS 48. OHSU. (2021). OHSU adjusts low-barrier test sites for COVID-19 First two hours daily will be reserved for priority groups. Retrieved from OHSU Social Hub website: NS -

49. Page, K. R., \& Flores-Miller, A. (2020). Lessons We've Learned - Covid-19 and the Undocumented Latinx Community. New England Journal of 

model for severe sepsis in resource-limited countries. Journal of Critical Care, 30(4), 861.e9-861.e14. https://doi.org/https://doi.org/10.1016/j.jcrc.2015.04.003

51. Press, A. (2021). Latino, Black neighborhoods struggle with COVID-19 test disparities. Retrieved from VOA News website: NS -

52. Rader, B., Astley, C. M., Sy, K. T. L., Sewalk, K., Hswen, Y., Brownstein, J. S., https://doi.org/10.1101/2020.04.25.20074419

53. Rajan, S., D. Cylus, J., \& Mckee, M. (2020). What do countries need to do to implement effective 'find, test, trace, isolate and support' systems? Journal of the Royal Society of Medicine, Vol. 113, pp. 245-250. https://doi.org/10.1177/0141076820939395

54. Safer, M. A., Tharps, Q. J., Jackson, T. C., \& Leventhal, H. (1979). Determinants of three stages of delay in seeking care at a medical clinic. Medical Care, 17(1), 11-29. https://doi.org/10.1097/00005650197901000-00002

55. Siegler, A. J., Hall, E., Luisi, N., Zlotorzynska, M., Wilde, G., Sanchez, T., ... Sullivan, P. S. (2020). Willingness to Seek Diagnostic Testing for SARS-CoV2 With Home, Drive-through, and Clinic-Based Specimen Collection Locations. Open Forum Infectious Diseases, 7(7 PG-ofaa269), ofaa269. 
https://doi.org/https://dx.doi.org/10.1093/ofid/ofaa269

746

747

748

749

750

751

752

753

754

755

756

757

758

759

760

761

762

763

764

765

766

767

768

56. Sotgiu, G., \& Dobler, C. C. (2020). Social stigma in the time of coronavirus disease 2019. European Respiratory Journal, 56(PG-23-25), 23-25. https://doi.org/10.1183/13993003.02461-2020

57. Thappa, P., \& Rana, K. (2020). Coronavirus Testing Hesitancy among Masses in India. International Journal of Health Sciences and Research (IJHSR), 10(June PG-139-141), 139-141. Retrieved from NS -

58. The Unity Council. (2020). Fruitvale Sanado Juntos Community COVID-19 Testing 9.26-9.27. 26-27. Retrieved from http://unitycouncil.org/wpcontent/uploads/2020/10/ExecSummaryFINAL-.pptx-2.pdf

59. Thunström, L., Ashworth, M., Shogren, J. F., Newbold, S., \& Finnoff, D. (2020). Testing for COVID-19: willful ignorance or selfless behavior? Behavioural Public Policy, (April PG-1-18), 1-18.

https://doi.org/10.1017/bpp.2020.15

60. Tricco, A. C., Lillie, E., Zarin, W., O’Brien, K. K., Colquhoun, H., Levac, D., ... Straus, S. E. (2018). PRISMA extension for scoping reviews (PRISMA-ScR): Checklist and explanation. Annals of Internal Medicine, 169(7), 467-473. https://doi.org/10.7326/M18-0850

61. Tsuyuki, R. T., \& Watson, K. E. (2020). COVID-19 testing by pharmacists. Canadian Pharmacists Journal, Vol. 153, pp. 314-315. https://doi.org/10.1177/1715163520961981

62. UNICEF. (2020). Social Stigma associated with COVID-19 A guide to preventing and addressing. Retrieved from NS -

63. WHO. (2020, July 31). Coronavirus: Events as they happen. Retrieved May 
25, 2021, from World Health Organisation website: https://www.who.int/emergencies/diseases/novel-coronavirus2019/events-as-they-happen

64. WHO. (2021). WHO Coronavirus Disease (COVID-19) Dashboard With Vaccination Data | WHO Coronavirus (COVID-19) Dashboard With Vaccination Data. Retrieved May 25, 2021, from World Health Organization website: https://covid19.who.int/ 65. Zimba, R., Kulkarni, S., Berry, A., You, W., Mirzayi, C., Westmoreland, D., ... Nash, D. (2020). Testing, Testing: What SARS-CoV-2 testing services do adults in the United States actually want? MedRxiv, (PG2020.09.15.20195180), 2020.09.15.20195180. 
794 Declarations

$795 \quad$ Funding

796 Funding was provided by Health Canada and the SPOR Evidence Alliance.

797

798 Competing interests/Conflicts of interest

799 The authors have no competing interests or conflicts of interest to declare.

800

801 Ethics Approval

802 Not applicable

803

804 Consent for publication

805 Not applicable

806

807 Availability of data and materials

808 The data supporting the conclusion of this article is available upon reasonable

809 request and the completion of a data transfer agreement.

810

811 Authors' Contributions

812 Each author contributed to the conception, design, collection of data, analysis

813 and write-up of the research article. With the exceptions of Dr. Andrea Triccio

814 and Dr. Janet Curran, who contributed to the conception, design, and write up of

815 the research article.

816 


\section{Acknowledgements}

818 We wish to thank Health Canada and the SPOR Evidence Alliance for their

819 funding that made this work possible. We also wish to thank the authors

820 referenced in this review for their hard work and commitment to

821 understanding and exploring COVID-19 testing hesitancy. Finally, we would

822 like to thank frontlines workers and their efforts to promote and provide

823 testing for all.

824 
825 Table 1. Examples of barriers to COVID-19 testing organized by type of delay.

\begin{tabular}{lll}
\hline Planning barriers & Process barriers & Outcomes
\end{tabular}

barriers

- Cost of Testing

- Health literacy

- Misinformation

- Testing criteria

(changes in testing criteria)

- Health status

- Trust in health system
- Availability of testing sites

- Waiting times (availability of human resources, testing supplies)

- Infrastructure features of testing sites

- Time delay in results (including laboratory capacity)

- Test properties (including pain, length of test)

- Test accuracy/sensitivity (false positives)

- Safety of test site (chance of infection)

- Trust in the process
- Stigma

- Personal cost

(cost of

isolation,

positive test

results: work, cost related to care etc.)

- Conseque nces on employme nt

- Health conseque nces 
829 Table 2. Strategies to address COVID-19 testing barriers and/or COVID-19 testing

830 hesitancy

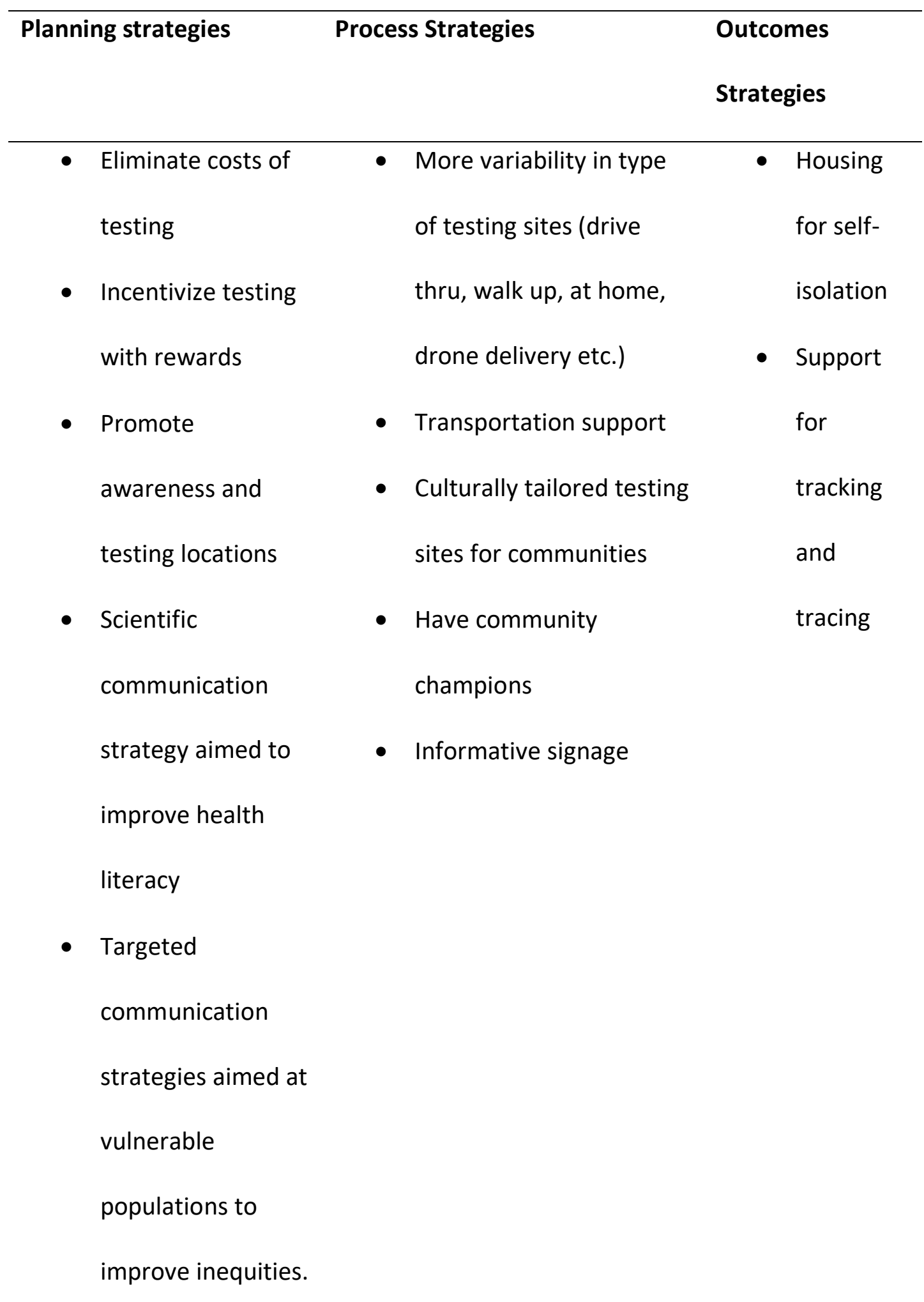




\section{Supplementary Files}

This is a list of supplementary files associated with this preprint. Click to download.

- supplementaryfiles.docx 